\title{
Some New Generalized Integral Inequalities for GA-s-Convex Functions via Hadamard Fractional Integrals
}

\author{
İmdat İşcan ${ }^{1}$ and Mustafa Aydin ${ }^{2}$ \\ ${ }^{1}$ Department of Mathematics, Faculty of Sciences and Arts, Giresun University, 28200 Giresun, Turkey \\ ${ }^{2}$ Department of Finance-Banking and Insurance, Alucra Turan Barutçu Vocational School, Giresun University, \\ Alucra, 28700 Giresun, Turkey
}

Correspondence should be addressed to İmdat İşcan; imdat.iscan@giresun.edu.tr

Received 22 April 2016; Revised 14 July 2016; Accepted 1 August 2016

Academic Editor: Chang-Jian Zhao

Copyright (C) 2016 İ Isşcan and M. Aydin. This is an open access article distributed under the Creative Commons Attribution License, which permits unrestricted use, distribution, and reproduction in any medium, provided the original work is properly cited.

We prove new generalization of Hadamard, Ostrowski, and Simpson inequalities in the framework of GA-s-convex functions and Hadamard fractional integral.

\section{Introduction}

Let a real function $f$ be defined on a nonempty interval $I$ of real line $\mathbb{R}$. The function $f$ is said to be convex on $I$ if inequality

$$
f(t x+(1-t) y) \leq t f(x)+(1-t) f(y)
$$

holds for all $x, y \in I$ and $t \in[0,1]$.

In [1], Breckner introduced $s$-convex functions as a generalization of convex functions as follows.

Definition 1. Let $s \in(0,1]$ be a fixed real number. A function $f:[0, \infty) \rightarrow[0, \infty)$ is said to be $s$-convex (in the second sense), or that $f$ belongs to the class $K_{s}^{2}$, if

$$
f(t x+(1-t) y) \leq t^{s} f(x)+(1-t)^{s} f(y)
$$

for all $x, y \in[0, \infty)$ and $t \in[0,1]$.

Of course, $s$-convexity means just convexity when $s=1$.

The following inequalities are well known in the literature as Hermite-Hadamard inequality, Ostrowski inequality, and Simpson inequality, respectively.
Theorem 2. Let $f: I \subseteq \mathbb{R} \rightarrow \mathbb{R}$ be a convex function defined on the interval I of real numbers and $a, b \in I$ with $a<b$. The following double inequality holds:

$$
f\left(\frac{a+b}{2}\right) \leq \frac{1}{b-a} \int_{a}^{b} f(x) d x \leq \frac{f(a)+f(b)}{2} .
$$

Theorem 3. Let $f: I \subseteq \mathbb{R} \rightarrow \mathbb{R}$ be a mapping differentiable in $I^{\circ}$, the interior of I, and let $a, b \in I^{\circ}$ with $a<b$. If $\left|f^{\prime}(x)\right| \leq M$, $x \in[a, b]$, then the following inequality holds:

$$
\begin{aligned}
& \left|f(x)-\frac{1}{b-a} \int_{a}^{b} f(t) d t\right| \\
& \quad \leq \frac{M}{b-a}\left[\frac{(x-a)^{2}+(b-x)^{2}}{2}\right]
\end{aligned}
$$

for all $x \in[a, b]$.

Theorem 4. Let $f:[a, b] \rightarrow \mathbb{R}$ be a four times' continuously differentiable mapping on $(a, b)$ and $\left\|f^{(4)}\right\|_{\infty}=$ $\sup _{x \in(a, b)}\left|f^{(4)}(x)\right|<\infty$. Then the following inequality holds:

$$
\begin{aligned}
\mid \frac{1}{3} & {\left[\frac{f(a)+f(b)}{2}+2 f\left(\frac{a+b}{2}\right)\right] } \\
- & \frac{1}{b-a} \int_{a}^{b} f(x) d x \mid \leq \frac{1}{2880}\left\|f^{(4)}\right\|_{\infty}(b-a)^{4} .
\end{aligned}
$$


We will give definitions of the right and left hand side Hadamard fractional integrals which are used throughout this paper.

Definition 5. Let $f \in L[a, b]$. The right-sided and left-sided Hadamard fractional integrals $J_{a+}^{\alpha} f$ and $J_{b-}^{\alpha} f$ of order $\alpha>0$ with $b>a \geq 0$ are defined by

$$
\begin{aligned}
& J_{a+}^{\alpha} f(x)=\frac{1}{\Gamma(\alpha)} \int_{a}^{x}\left(\ln \frac{x}{t}\right)^{\alpha-1} f(t) \frac{d t}{t}, \quad a<x<b, \\
& J_{b-}^{\alpha} f(x)=\frac{1}{\Gamma(\alpha)} \int_{x}^{b}\left(\ln \frac{t}{x}\right)^{\alpha-1} f(t) \frac{d t}{t}, \quad a<x<b,
\end{aligned}
$$

respectively, where $\Gamma(\alpha)$ is the Gamma function defined by $\Gamma(\alpha)=\int_{0}^{\infty} e^{-t} t^{\alpha-1} d t$ (see [2]).

In recent years, many authors have studied errors estimations for Hermite-Hadamard, Ostrowski, and Simpson inequalities; for refinements, counterparts, and generalization see [3-10].

Definition 6 (see $[11,12])$. A function $f: I \subseteq(0, \infty) \rightarrow \mathbb{R}$ is said to be GA-convex (geometric-arithmetically convex) if

$$
f\left(x^{t} y^{1-t}\right) \leq t f(x)+(1-t) f(y)
$$

for all $x, y \in I$ and $t \in[0,1]$.

Definition 7 (see [13]). For $s \in(0,1]$, a function $f$ : $I \subseteq(0, \infty) \rightarrow \mathbb{R}$ is said to be GA-s-convex (geometricarithmetically $s$-convex) if

$$
f\left(x^{t} y^{1-t}\right) \leq t^{s} f(x)+(1-t)^{s} f(y)
$$

for all $x, y \in I$ and $t \in[0,1]$.

It can be easily seen that if $s=1, \mathrm{GA}$-s-convexity reduces to GA-convexity.

For recent results and generalizations concerning GAconvex and GA-s-convex functions see [13-19].

Lemma 8 (see [20]). For $\alpha>0$ and $\mu>0$, one has

$$
\int_{0}^{1} t^{\alpha-1} \mu^{t} d t=\mu \sum_{k=1}^{\infty} \frac{(-1)^{k-1}(\ln \mu)^{k-1}}{(\alpha)_{k}}<\infty
$$

where

$$
(\alpha)_{k}=\alpha(\alpha+1)(\alpha+2) \cdots(\alpha+k-1) .
$$

Let $f: I \subseteq(0, \infty) \rightarrow \mathbb{R}$ be a differentiable function on $I^{\circ}$, the interior of $I$; in sequel of this paper we will take

$$
\begin{aligned}
I_{f}(x, \lambda, \alpha, a, b)= & (1-\lambda)\left[\ln ^{\alpha} \frac{x}{a}+\ln ^{\alpha} \frac{b}{x}\right] f(x) \\
& +\lambda\left[f(a) \ln ^{\alpha} \frac{x}{a}+f(b) \ln ^{\alpha} \frac{b}{x}\right] \\
& -\Gamma(\alpha+1)\left[J_{x-}^{\alpha} f(a)+J_{x+}^{\alpha} f(b)\right]
\end{aligned}
$$

where $a, b \in I$ with $a<b, x \in[a, b], \lambda \in[0,1], \alpha>0$, and $\Gamma$ is Euler Gamma function.

In [21], İşcan gave Hermite-Hadamard's inequalities for GA-convex functions in fractional integral forms as follows.

Theorem 9. Let $f: I \subseteq(0, \infty) \rightarrow \mathbb{R}$ be a function such that $f \in L[a, b]$, where $a, b \in I$ with $a<b$. If $f$ is a GA-convex function on $[a, b]$, then the following inequalities for fractional integrals hold:

$$
\begin{aligned}
f(\sqrt{a b}) & \leq \frac{\Gamma(\alpha+1)}{2(\ln (b / a))^{\alpha}}\left\{J_{a+}^{\alpha} f(b)+J_{b-}^{\alpha} f(a)\right\} \\
& \leq \frac{f(a)+f(b)}{2}
\end{aligned}
$$

with $\alpha>0$.

In [21], İşcan obtained some new inequalities for quasigeometrically convex functions via fractional integrals by using the following lemma.

Lemma 10. Let $f: I \subseteq(0, \infty) \rightarrow \mathbb{R}$ be a differentiable function on $I^{\circ}$ such that $f^{\prime} \in L[a, b]$, where $a, b \in I$ with $a<b$. Then for all $x \in[a, b], \lambda \in[0,1]$, and $\alpha>0$ one has

$$
\begin{aligned}
I_{f}(x, \lambda, \alpha, a, b) \\
=a\left(\ln \frac{x}{a}\right)^{\alpha+1} \int_{0}^{1}\left(t^{\alpha}-\lambda\right)\left(\frac{x}{a}\right)^{t} f^{\prime}\left(x^{t} a^{1-t}\right) d t \\
\quad-b\left(\ln \frac{b}{x}\right)^{\alpha+1} \int_{0}^{1}\left(t^{\alpha}-\lambda\right)\left(\frac{x}{b}\right)^{t} f^{\prime}\left(x^{t} b^{1-t}\right) d t .
\end{aligned}
$$

In this paper, we will use Lemma 10 to obtain some new inequalities on generalization of Hadamard, Ostrowski, and Simpson type inequalities for GA-s-convex functions via Hadamard fractional integral.

\section{Generalized Integral Inequalities for Some GA-s-Convex Functions via Fractional Integrals}

Theorem 11. Let $f: I \subset(0, \infty) \rightarrow \mathbb{R}$ be a differentiable function on $I^{\circ}$ such that $f^{\prime} \in L[a, b]$, where $a, b \in I^{\circ}$ with $a<b$. If $\left|f^{\prime}\right|^{q}$ is GA-s-convex on $[a, b]$ in the second sense for some fixed $q \geq 1, x \in[a, b], \lambda \in[0,1]$, and $\alpha>0$ then the following inequality for fractional integrals holds:

$$
\begin{aligned}
& \left|I_{f}(x, \lambda, \alpha, a, b)\right| \leq A_{1}^{1-1 / q}(\alpha, \lambda)\left\{a\left(\ln \frac{x}{a}\right)^{\alpha+1}\right. \\
& \cdot\left(\left|f^{\prime}(x)\right|^{q} A_{2}\left(\left(\frac{x}{a}\right)^{q}, \alpha, \lambda, s\right)\right. \\
& \left.+\left|f^{\prime}(a)\right|^{q} A_{3}\left(\left(\frac{x}{a}\right)^{q}, \alpha, \lambda, s\right)\right)^{1 / q}+b\left(\ln \frac{b}{x}\right)^{\alpha+1}
\end{aligned}
$$




$$
\begin{aligned}
& \cdot\left(\left|f^{\prime}(x)\right|^{q} A_{2}\left(\left(\frac{x}{b}\right)^{q}, \alpha, \lambda, s\right)\right. \\
& \left.\left.+\left|f^{\prime}(b)\right|^{q} A_{3}\left(\left(\frac{x}{b}\right)^{q}, \alpha, \lambda, s\right)\right)^{1 / q}\right\},
\end{aligned}
$$

where

$$
\begin{aligned}
& A_{1}(\alpha, \lambda)=\frac{2 \alpha \lambda^{1+1 / \alpha}+1}{\alpha+1}-\lambda, \\
& A_{2}\left(\left(\frac{x}{u}\right)^{q}, \alpha, \lambda, s\right)=\int_{0}^{1}\left|t^{\alpha}-\lambda\right|\left(\frac{x}{u}\right)^{q t} t^{s} d t, \\
& A_{3}\left(\left(\frac{x}{u}\right)^{q}, \alpha, \lambda, s\right)=\int_{0}^{1}\left|t^{\alpha}-\lambda\right|\left(\frac{x}{u}\right)^{q t}(1-t)^{s} d t, \\
& u=a, b .
\end{aligned}
$$

Proof. Using Lemma 10, property of the modulus, and the power-mean inequality, we have

$$
\begin{aligned}
& \left|I_{f}(x, \lambda, \alpha, a, b)\right| \leq a\left(\ln \frac{x}{a}\right)^{\alpha+1} \\
& \cdot \int_{0}^{1}\left|t^{\alpha}-\lambda\right|\left(\frac{x}{a}\right)^{t}\left|f^{\prime}\left(x^{t} a^{1-t}\right)\right| d t+b\left(\ln \frac{b}{x}\right)^{\alpha+1} \\
& \cdot \int_{0}^{1}\left|t^{\alpha}-\lambda\right|\left(\frac{x}{b}\right)^{t}\left|f^{\prime}\left(x^{t} b^{1-t}\right)\right| d t \leq a\left(\ln \frac{x}{a}\right)^{\alpha+1} \\
& \cdot\left(\int_{0}^{1}\left|t^{\alpha}-\lambda\right| d t\right)^{1-1 / q} \\
& \cdot\left(\int_{0}^{1}\left|t^{\alpha}-\lambda\right|\left(\frac{x}{a}\right)^{q t}\left|f^{\prime}\left(x^{t} a^{1-t}\right)\right|^{q} d t\right)^{1 / q} \\
& +b\left(\ln \frac{b}{x}\right)^{\alpha+1}\left(\int_{0}^{1}\left|t^{\alpha}-\lambda\right| d t\right){ }^{1-1 / q} \\
& \quad\left(\int_{0}^{1}\left|t^{\alpha}-\lambda\right|\left(\frac{x}{b}\right)^{q t}\left|f^{\prime}\left(x^{t} b^{1-t}\right)\right|^{q} d t\right)^{1 / q} \cdot
\end{aligned}
$$

Since $\left|f^{\prime}\right|^{q}$ is GA-s-convex on $[a, b]$, we get

$$
\begin{aligned}
& \int_{0}^{1}\left|t^{\alpha}-\lambda\right|\left(\frac{x}{a}\right)^{q t}\left|f^{\prime}\left(x^{t} a^{1-t}\right)\right|^{q} d t \leq \int_{0}^{1}\left|t^{\alpha}-\lambda\right| \\
& \cdot\left(\frac{x}{a}\right)^{q t}\left(t^{s}\left|f^{\prime}(x)\right|^{q}+(1-t)^{s}\left|f^{\prime}(a)\right|^{q}\right) d t \\
& =\left|f^{\prime}(x)\right|^{q} A_{2}\left(\frac{x}{a}, \alpha, \lambda, s, q\right)+\left|f^{\prime}(a)\right|^{q} \\
& \cdot A_{3}\left(\frac{x}{a}, \alpha, \lambda, s, q\right),
\end{aligned}
$$

$$
\begin{aligned}
& \int_{0}^{1}\left|t^{\alpha}-\lambda\right|\left(\frac{x}{b}\right)^{q t}\left|f^{\prime}\left(x^{t} b^{1-t}\right)\right|^{q} d t \leq \int_{0}^{1}\left|t^{\alpha}-\lambda\right| \\
& \cdot\left(\frac{x}{b}\right)^{q t}\left(t^{s}\left|f^{\prime}(x)\right|^{q}+(1-t)^{s}\left|f^{\prime}(b)\right|^{q}\right) d t \\
& =\left|f^{\prime}(x)\right|^{q} A_{2}\left(\frac{x}{b}, \alpha, \lambda, s, q\right)+\left|f^{\prime}(b)\right|^{q} \\
& \cdot A_{3}\left(\frac{x}{b}, \alpha, \lambda, s, q\right)
\end{aligned}
$$

and by a simple computation, we have

$$
\begin{aligned}
\int_{0}^{1}\left|t^{\alpha}-\lambda\right| d t & =\int_{0}^{\lambda^{1 / \alpha}}\left(\lambda-t^{\alpha}\right) d t+\int_{\lambda^{1 / \alpha}}^{1}\left(t^{\alpha}-\lambda\right) d t \\
& =\frac{2 \alpha \lambda^{1+1 / \alpha}+1}{\alpha+1}-\lambda .
\end{aligned}
$$

Hence, If we use (18), (19), and (20) in (17), we obtain the desired result. This completes the proof.

Corollary 12. Under the assumptions of Theorem 11 with $s=$ 1, inequality (15) reduces to the following inequality:

$$
\begin{aligned}
& \left|I_{f}(x, \lambda, \alpha, a, b)\right| \leq A_{1}^{1-1 / q}(\alpha, \lambda)\left\{a\left(\ln \frac{x}{a}\right)^{\alpha+1}\right. \\
& \cdot\left(\left|f^{\prime}(x)\right|^{q} A_{2}\left(\left(\frac{x}{a}\right)^{q}, \alpha, \lambda, 1\right)\right. \\
& \left.+\left|f^{\prime}(a)\right|^{q} A_{3}\left(\left(\frac{x}{a}\right)^{q}, \alpha, \lambda, 1\right)\right)^{1 / q}+b\left(\ln \frac{b}{x}\right)^{\alpha+1} \\
& \cdot\left(\left|f^{\prime}(x)\right|^{q} A_{2}\left(\left(\frac{x}{b}\right)^{q}, \alpha, \lambda, 1\right)\right. \\
& \left.\left.+\left|f^{\prime}(b)\right|^{q} A_{3}\left(\left(\frac{x}{b}\right)^{q}, \alpha, \lambda, 1\right)\right)^{1 / q}\right\} .
\end{aligned}
$$

Corollary 13. Under the assumptions of Theorem 11 with $s=1$ and $\alpha=1$, inequality (15) reduces to the following inequality:

$$
\begin{aligned}
& \left(\ln \frac{b}{a}\right)^{-1}\left|I_{f}(x, \lambda, 1, a, b)\right|=\mid(1-\lambda) f(x) \\
& +\lambda\left[\frac{f(a) \ln (x / a)+f(b) \ln (b / x)}{\ln (b / a)}\right]-\frac{1}{\ln (b / a)} \\
& \cdot \int_{a}^{b} \frac{f(u)}{u} d u \mid \leq\left(\ln \frac{b}{a}\right)^{-1} A_{1}^{1-1 / q}(1, \lambda)\left\{a\left(\ln \frac{x}{a}\right)^{2}\right. \\
& \cdot\left(\left|f^{\prime}(x)\right|^{q} A_{2}\left(\mu_{a}, 1, \lambda, 1\right)\right. \\
& \left.+\left|f^{\prime}(a)\right|^{q} A_{3}\left(\mu_{a}, 1, \lambda, 1\right)\right)^{1 / q}+b\left(\ln \frac{b}{x}\right)^{2} \\
& \cdot\left(\left|f^{\prime}(x)\right|^{q} A_{2}\left(\mu_{b}, 1, \lambda, 1\right)\right. \\
& \left.\left.+\left|f^{\prime}(b)\right|^{q} A_{3}\left(\mu_{b}, 1, \lambda, 1\right)\right)^{1 / q}\right\},
\end{aligned}
$$

where 


$$
\begin{aligned}
A_{1}(1, \lambda) & =\frac{\left(2 \lambda^{2}-2 \lambda+1\right)}{2}, \\
A_{2}\left(\mu_{u}, 1, \lambda, 1\right) & =\frac{\left\{\left(\mu_{u}-2 \lambda^{2} \mu_{u}^{\lambda}\right) \ln ^{2} \mu_{u}+\left(\lambda \mu_{u}^{\lambda} \ln \mu_{u}-\mu_{u}^{\lambda}+1\right)\left(\lambda \ln \mu_{u}+\lambda+4\right)-(\lambda+2)\left(\mu_{u} \ln \mu_{u}-\mu_{u}+1\right)\right\}}{\left(\ln \mu_{u}\right)^{3}} \\
A_{3}\left(\mu_{u}, 1, \lambda, 1\right) & =\frac{\left[2 \mu_{u}^{\lambda}+\mu_{u} \ln \mu_{u}-\lambda\left(1+\mu_{u}\right) \ln \mu_{u}-\mu_{u}-1\right]}{\left(\ln \mu_{u}\right)^{2}}-A_{2}\left(\mu_{u}, 1, \lambda, 1\right), \\
\mu_{u} & =\left(\frac{x}{u}\right)^{q}, \quad u=a, b .
\end{aligned}
$$

Corollary 14. Under the assumptions of Theorem 11 with $q=$ 1, inequality (15) reduces to the following inequality:

$$
\begin{aligned}
& \left|I_{f}(x, \lambda, \alpha, a, b)\right| \leq\left\{a\left(\ln \frac{x}{a}\right)^{\alpha+1}\right. \\
& \cdot\left(\left|f^{\prime}(x)\right| A_{2}\left(\frac{x}{a}, \alpha, \lambda, s\right)\right. \\
& \left.+\left|f^{\prime}(a)\right| A_{3}\left(\frac{x}{a}, \alpha, \lambda, s\right)\right)+b\left(\ln \frac{b}{x}\right)^{\alpha+1} \\
& \cdot\left(\left|f^{\prime}(x)\right| A_{2}\left(\frac{x}{b}, \alpha, \lambda, s\right)\right. \\
& \left.\left.+\left|f^{\prime}(b)\right| A_{3}\left(\frac{x}{b}, \alpha, \lambda, s\right)\right)\right\} .
\end{aligned}
$$

Corollary 15. Under the assumptions of Theorem 11 with $x=$ $\sqrt{a b}, \lambda=1 / 3$, from inequality (15), one gets the following Simpson type inequality for fractional integrals:

$$
\begin{aligned}
& \left|2^{\alpha-1}\left(\ln \frac{b}{a}\right)^{-\alpha} I_{f}\left(\sqrt{a b}, \frac{1}{3}, \alpha, a, b\right)\right|=\mid \frac{1}{6}[f(a) \\
& +4 f(\sqrt{a b})+f(b)]-\frac{2^{\alpha-1} \Gamma(\alpha+1)}{(\ln (b / a))^{\alpha}}\left[J_{\sqrt{a b}-}^{\alpha} f(a)\right. \\
& \left.+J_{\sqrt{a b}+}^{\alpha} f(b)\right] \mid \leq \frac{\ln (b / a)}{4} A_{1}^{1-1 / q}\left(\alpha, \frac{1}{3}\right) \\
& +\left\{a \left(\left|f^{\prime}(\sqrt{a b})\right|^{q} A_{2}\left(\left(\frac{b}{a}\right)^{q / 2}, \alpha, \frac{1}{3}, s\right)\right.\right. \\
& \left.+\left|f^{\prime}(a)\right|^{q} A_{3}\left(\left(\frac{b}{a}\right)^{q / 2}, \alpha, \frac{1}{3}, s\right)\right)^{1 / q} \\
& +b\left(\left|f^{\prime}(\sqrt{a b})\right|^{q} A_{2}\left(\left(\frac{a}{b}\right)^{q / 2}, \alpha, \frac{1}{3}, s\right)\right. \\
& \left.\left.+\left|f^{\prime}(b)\right|^{q} A_{3}\left(\left(\frac{a}{b}\right)^{q / 2}, \alpha, \frac{1}{3}, s\right)\right)^{1 / q}\right\} .
\end{aligned}
$$

Corollary 16. Under the assumptions of Theorem 11 with $x=$ $\sqrt{a b}, \lambda=0$, from inequality (15), one gets

$$
\begin{aligned}
& \left|2^{\alpha-1}\left(\ln \frac{b}{a}\right)^{-\alpha} I_{f}(\sqrt{a b}, 0, \alpha, a, b)\right|=\mid f(\sqrt{a b}) \\
& -\frac{2^{\alpha-1} \Gamma(\alpha+1)}{(\ln (b / a))^{\alpha}}\left[J_{\sqrt{a b}-}^{\alpha} f(a)+J_{\sqrt{a b}+}^{\alpha} f(b)\right] \mid \\
& \leq \frac{\ln (b / a)}{4}\left(\frac{1}{\alpha+1}\right)^{1-1 / q} \\
& .\left\{a \left(\left|f^{\prime}(\sqrt{a b})\right|^{q} A_{2}\left(\left(\frac{b}{a}\right)^{q / 2}, \alpha, 0, s\right)\right.\right. \\
& \left.+\left|f^{\prime}(a)\right|^{q} A_{3}\left(\left(\frac{b}{a}\right)^{q / 2}, \alpha, 0, s\right)\right)^{1 / q} \\
& +b\left(\left|f^{\prime}(\sqrt{a b})\right|^{q} A_{2}\left(\left(\frac{a}{b}\right)^{q / 2}, \alpha, 0, s\right)\right. \\
& \left.\left.+\left|f^{\prime}(b)\right|^{q} A_{3}\left(\left(\frac{a}{b}\right)^{q / 2}, \alpha, 0, s\right)\right)^{1 / q}\right\} .
\end{aligned}
$$

Corollary 17. Under the assumptions of Theorem 11 with $x=$ $\sqrt{a b}$ and $\lambda=1$, from inequality (15) one gets

$$
\begin{aligned}
& \left|2^{\alpha-1}\left(\ln \frac{b}{a}\right)^{-\alpha} I_{f}(\sqrt{a b}, 1, \alpha, a, b)\right|=\mid \frac{f(a)+f(b)}{2} \\
& -\frac{2^{\alpha-1} \Gamma(\alpha+1)}{(\ln (b / a))^{\alpha}}\left[J_{\sqrt{a b}-}^{\alpha} f(a)+J_{\sqrt{a b}+}^{\alpha} f(b)\right] \mid \\
& \leq \frac{\ln (b / a)}{4}\left(\frac{\alpha}{\alpha+1}\right)^{1-1 / q} \\
& .\left\{a \left[\left|f^{\prime}(\sqrt{a b})\right|^{q} A_{2}\left(\left(\frac{b}{a}\right)^{q / 2}, \alpha, 1, s\right)\right.\right. \\
& \left.+\left|f^{\prime}(a)\right|^{q} A_{3}\left(\left(\frac{b}{a}\right)^{q / 2}, \alpha, 1, s\right)\right]^{1 / q}
\end{aligned}
$$


Chinese Journal of Mathematics

5

$$
\begin{aligned}
& +b\left[\left|f^{\prime}(\sqrt{a b})\right|^{q} A_{2}\left(\left(\frac{a}{b}\right)^{q / 2}, \alpha, 1, s\right)\right. \\
& \left.\left.+\left|f^{\prime}(b)\right|^{q} A_{3}\left(\left(\frac{a}{b}\right)^{q / 2}, \alpha, 1, s\right)\right]^{1 / q}\right\} .
\end{aligned}
$$

$$
\left.\begin{array}{l}
\cdot\left[A_{2}\left(\left(\frac{a}{b}\right)^{q / 2}, \alpha, 0, s\right)\right. \\
\left.+A_{3}\left(\left(\frac{a}{b}\right)^{q / 2}, \alpha, 0, s\right)\right]^{1 / q}
\end{array}\right\}
$$

(27)

for all $x \in[a, b]$.

Theorem 19. Let $f: I \subset(0, \infty) \rightarrow \mathbb{R}$ be a differentiable funcion on $I^{\circ}$ such that $f^{\prime} \in L[a, b]$, where $a, b \in I^{\circ}$ with $a<b$. If $\left|f^{\prime}\right|^{q}$ is GA-s-convex on $[a, b]$ for some fixed $q>1, x \in[a, b]$, $\lambda \in[0,1]$, and $\alpha>0$ then the following inequality for fractional integrals holds:

$$
\begin{aligned}
& \left|I_{f}(x, \lambda, \alpha, a, b)\right| \leq C_{1}^{1 / p}(\alpha, \lambda)\left\{a\left(\ln \frac{x}{a}\right)^{\alpha+1}\right. \\
& \cdot\left(\left|f^{\prime}(x)\right|^{q} C_{2}\left(\left(\frac{x}{a}\right)^{q}, s\right)\right. \\
& \left.+\left|f^{\prime}(a)\right|^{q} C_{3}\left(\left(\frac{x}{a}\right)^{q}, s\right)\right)^{1 / q}+b\left(\ln \frac{b}{x}\right)^{\alpha+1} \\
& \cdot\left(\left|f^{\prime}(x)\right|^{q} C_{2}\left(\left(\frac{x}{b}\right)^{q}, s\right)\right. \\
& \left.\left.+\left|f^{\prime}(b)\right|^{q} C_{3}\left(\left(\frac{x}{b}\right)^{q}, s\right)\right)^{1 / q}\right\},
\end{aligned}
$$

where $1 / p+1 / q=1$ and

$$
\begin{aligned}
& C_{1}(\alpha, \lambda)= \begin{cases}\frac{1}{(\alpha p+1)}, & \lambda=0 \\
\frac{\lambda^{1+p+1 / \alpha}}{\alpha} \beta\left(\frac{1}{\alpha}, p+1\right)+\frac{(1-\lambda)^{p+1}}{\alpha(p+1)} \cdot{ }_{2} F_{1}\left(1-\frac{1}{\alpha}, 1 ; p+2 ; 1-\lambda\right), & 0<\lambda \leq 1,\end{cases} \\
& C_{2}\left(\left(\frac{x}{u}\right)^{q}, s\right)=\left(\frac{x}{u}\right)^{q} \sum_{k=1}^{\infty} \frac{(-1)^{k-1}\left(\ln (x / u)^{q}\right)^{k-1}}{(s+1)_{k}}, \\
& C_{3}\left(\left(\frac{x}{u}\right)^{q}, s\right)=\sum_{k=1}^{\infty} \frac{(-1)^{k-1}\left(-\ln (x / u)^{q}\right)^{k-1}}{(s+1)_{k}}, \quad u=a, b .
\end{aligned}
$$

Proof. Using Lemma 10, property of the modulus, the Hölder inequality, and GA-s-convexity of $\left|f^{\prime}\right|^{q}$, we have

$$
\begin{aligned}
& \left|I_{f}(x, \lambda, \alpha, a, b)\right| \leq a\left(\ln \frac{x}{a}\right)^{\alpha+1} \int_{0}^{1}\left|t^{\alpha}-\lambda\right|\left(\frac{x}{a}\right)^{t} \\
& \cdot\left|f^{\prime}\left(x^{t} a^{1-t}\right)\right| d t+b\left(\ln \frac{b}{x}\right)^{\alpha+1} \int_{0}^{1}\left|t^{\alpha}-\lambda\right| \\
& \cdot\left(\frac{x}{b}\right)^{t}\left|f^{\prime}\left(x^{t} b^{1-t}\right)\right| d t \leq a\left(\ln \frac{x}{a}\right)^{\alpha+1} \\
& \cdot\left(\int_{0}^{1}\left|t^{\alpha}-\lambda\right|^{p} d t\right)^{1 / p}
\end{aligned}
$$

$$
\begin{aligned}
& \cdot\left(\int_{0}^{1}\left(\frac{x}{a}\right)^{q t}\left|f^{\prime}\left(x^{t} a^{1-t}\right)\right|^{q} d t\right)^{1 / q}+b\left(\ln \frac{b}{x}\right)^{\alpha+1} \\
& \cdot\left(\int_{0}^{1}\left|t^{\alpha}-\lambda\right|^{p} d t\right)^{1 / p} \\
& \cdot\left(\int_{0}^{1}\left(\frac{x}{b}\right)^{q t}\left|f^{\prime}\left(x^{t} b^{1-t}\right)\right|^{q} d t\right)^{1 / q} \\
& \leq\left(\int_{0}^{1}\left|t^{\alpha}-\lambda\right|^{p} d t\right)^{1 / p}\left\{a\left(\ln \frac{x}{a}\right)^{\alpha+1}\right. \\
& \cdot\left(\left|f^{\prime}(x)\right|^{q} \int_{0}^{1} \mu_{a}^{t} t^{s}+\left|f^{\prime}(a)\right|^{q} \int_{0}^{1} \mu_{a}^{t}(1-t)^{s} d t\right)^{1 / q}
\end{aligned}
$$


6

Chinese Journal of Mathematics

$$
\begin{aligned}
& +b\left(\ln \frac{b}{x}\right)^{\alpha+1}\left(\left|f^{\prime}(x)\right|^{q} \int_{0}^{1} \mu_{b}^{t} t^{s}\right. \\
& \left.\left.+\left|f^{\prime}(b)\right|^{q} \int_{0}^{1} \mu_{b}^{t}(1-t)^{s} d t\right)^{1 / q}\right\},
\end{aligned}
$$

where $\mu_{a}=(x / a)^{q}, \mu_{b}=(x / b)^{q}$ and

$$
\begin{array}{rlrl}
\int_{0}^{1}\left|t^{\alpha}-\lambda\right|^{p} d t & =\int_{0}^{\lambda^{1 / \alpha}}\left(\lambda-t^{\alpha}\right)^{p} d t+\int_{\lambda^{1 / \alpha}}^{1}\left(t^{\alpha}-\lambda\right)^{p} d t & \lambda=0 \\
& = \begin{cases}\frac{1}{(\alpha p+1)}, & \\
\frac{\lambda^{(\alpha p+1) / \alpha}}{\alpha} \beta\left(\frac{1}{\alpha}, p+1\right)+\frac{(1-\lambda)^{p+1}}{\alpha(p+1)} \cdot{ }_{2} F_{1}\left(1-\frac{1}{\alpha}, 1 ; p+2 ; 1-\lambda\right), & 0<\lambda \leq 1 .\end{cases}
\end{array}
$$

Using Lemma 8, we have

$$
\begin{aligned}
\int_{0}^{1} \mu_{u}^{t} t^{s} d t & =\mu_{u} \sum_{k=1}^{\infty} \frac{(-1)^{k-1}\left(\ln \mu_{u}\right)^{k-1}}{(s+1)_{k}}, \\
\int_{0}^{1} \mu_{u}^{t}(1-t)^{s} d t & =\int_{0}^{1} \mu_{u}^{1-t} t^{s} d t \\
& =\sum_{k=1}^{\infty} \frac{(-1)^{k-1}\left(-\ln \mu_{u}\right)^{k-1}}{(s+1)_{k}}
\end{aligned}
$$

Hence, if we use (32)-(33) in (31) and replacing $\mu_{a}=(x / a)^{q}$, $\mu_{b}=(x / b)^{q}$, we obtain the desired result. This completes the proof.

Corollary 20. Under the assumptions of Theorem 19 with $s=$ 1, inequality (29) reduces to the following inequality:

$$
\begin{aligned}
& \left|I_{f}(x, \lambda, \alpha, a, b)\right| \leq C_{1}^{1 / p}(\alpha, \lambda)\left\{a\left(\ln \frac{x}{a}\right)^{\alpha+1}\right. \\
& \cdot\left(\left|f^{\prime}(x)\right|^{q} C_{2}\left(\left(\frac{x}{a}\right)^{q}, 1\right)\right. \\
& \left.+\left|f^{\prime}(a)\right|^{q} C_{3}\left(\left(\frac{x}{a}\right)^{q}, 1\right)\right)^{1 / q}+b\left(\ln \frac{b}{x}\right)^{\alpha+1} \\
& \cdot\left(\left|f^{\prime}(x)\right|^{q} C_{2}\left(\left(\frac{x}{b}\right)^{q}, 1\right)\right. \\
& \left.\left.+\left|f^{\prime}(b)\right|^{q} C_{3}\left(\left(\frac{x}{b}\right)^{q}, 1\right)\right)^{1 / q}\right\} .
\end{aligned}
$$

Corollary 21. Under the assumptions of Theorem 19 with $s=1$ and $\alpha=1$, inequality (29) reduces to the following inequality:

$$
\begin{aligned}
& \left|I_{f}(x, \lambda, 1, a, b)\right|=\mid \ln \frac{b}{a}(1-\lambda) f(x)+\lambda\left[f(a) \ln \frac{x}{a}\right. \\
& \left.+f(b) \ln \frac{b}{x}\right]-\int_{a}^{b} \frac{f(u)}{u} d u \mid \\
& \quad \leq\left(\frac{\lambda^{p+1}+(1-\lambda)^{p+1}}{p+1}\right)^{1 / p}\left\{a\left(\ln \frac{x}{a}\right)^{2}\right. \\
& +\left(\left|f^{\prime}(x)\right|^{q} C_{2}\left(\left(\frac{x}{a}\right)^{q}, 1\right)\right. \\
& \left.+\left|f^{\prime}(a)\right|^{q} C_{3}\left(\left(\frac{x}{a}\right)^{q}, 1\right)\right)^{1 / q}+b\left(\ln \frac{b}{x}\right)^{2} \\
& +\left(\left|f^{\prime}(x)\right|^{q} C_{2}\left(\left(\frac{x}{b}\right)^{q}, 1\right)\right. \\
& \left.\left.+\left|f^{\prime}(b)\right|^{q} C_{3}\left(\left(\frac{x}{b}\right)^{q}, 1\right)\right)^{1 / q}\right\} .
\end{aligned}
$$

Corollary 22. Under the assumptions of Theorem 19 with $x=$ $\sqrt{a b}, \lambda=1 / 3$, from inequality (29), one gets the following Simpson type inequality for fractional integrals:

$$
\begin{aligned}
& \left|2^{\alpha-1}\left(\ln \frac{b}{a}\right)^{-\alpha} I_{f}\left(\sqrt{a b}, \frac{1}{3}, \alpha, a, b\right)\right|=\mid \frac{1}{6}[f(a) \\
& +4 f(\sqrt{a b})+f(b)]-\frac{2^{\alpha-1} \Gamma(\alpha+1)}{(\ln (b / a))^{\alpha}}\left[J_{\sqrt{a b}-}^{\alpha} f(a)\right. \\
& \left.+J_{\sqrt{a b}+}^{\alpha} f(b)\right] \mid \leq \frac{\ln (b / a)}{4} C_{1}^{1 / p}\left(\alpha, \frac{1}{3}\right) \\
& \quad \cdot\left\{a \left(\left|f^{\prime}(\sqrt{a b})\right|^{q} C_{2}\left(\left(\frac{b}{a}\right)^{q / 2}, s\right)\right.\right.
\end{aligned}
$$




$$
\begin{aligned}
& \left.+\left|f^{\prime}(a)\right|^{q} C_{3}\left(\left(\frac{b}{a}\right)^{q / 2}, s\right)\right)^{1 / q} \\
& +b\left(\left|f^{\prime}(\sqrt{a b})\right|^{q} C_{2}\left(\left(\frac{a}{b}\right)^{q / 2}, s\right)\right. \\
& \left.\left.+\left|f^{\prime}(b)\right|^{q} C_{3}\left(\left(\frac{a}{b}\right)^{q / 2}, s\right)\right)^{1 / q}\right\} .
\end{aligned}
$$

Corollary 23. Under the assumptions of Theorem 19 with $x=$ $\sqrt{a b}, \lambda=0$, from inequality (29), one gets

$$
\begin{aligned}
& \left|2^{\alpha-1}\left(\ln \frac{b}{a}\right)^{-\alpha} I_{f}(\sqrt{a b}, 0, \alpha, a, b)\right|=\mid f(\sqrt{a b}) \\
& -\frac{2^{\alpha-1} \Gamma(\alpha+1)}{(\ln (b / a))^{\alpha}}\left[J_{\sqrt{a b}-}^{\alpha} f(a)+J_{\sqrt{a b}+}^{\alpha} f(b)\right] \mid \\
& \leq \frac{\ln (b / a)}{4}\left(\frac{1}{\alpha p+1}\right)^{1 / p} \\
& .\left\{a \left(\left|f^{\prime}(\sqrt{a b})\right|^{q} C_{2}\left(\left(\frac{b}{a}\right)^{q / 2}, s\right)\right.\right. \\
& \left.+\left|f^{\prime}(a)\right|^{q} C_{3}\left(\left(\frac{b}{a}\right)^{q / 2}, s\right)\right)^{1 / q} \\
& +b\left(\left|f^{\prime}(\sqrt{a b})\right|^{q} C_{2}\left(\left(\frac{a}{b}\right)^{q / 2}, s\right)\right. \\
& \left.\left.+\left|f^{\prime}(b)\right|^{q} C_{3}\left(\left(\frac{a}{b}\right)^{q / 2}, s\right)\right)^{1 / q}\right\} .
\end{aligned}
$$

Corollary 24. Under the assumptions of Theorem 19 with $x=\sqrt{a b}$ and $\lambda=1$, from inequality (29) one gets

$$
\begin{aligned}
& \left|2^{\alpha-1}\left(\ln \frac{b}{a}\right)^{-\alpha} I_{f}(\sqrt{a b}, 1, \alpha, a, b)\right|=\mid \frac{f(a)+f(b)}{2} \\
& -\frac{2^{\alpha-1} \Gamma(\alpha+1)}{(\ln (b / a))^{\alpha}}\left[J_{\sqrt{a b}-}^{\alpha} f(a)+J_{\sqrt{a b}+}^{\alpha} f(b)\right] \mid \\
& \leq \frac{\ln (b / a)}{4}\left(\frac{1}{\alpha} \beta\left(\frac{1}{\alpha}, p+1\right)\right)^{1 / p} \\
& +\left\{a \left[\left|f^{\prime}(\sqrt{a b})\right|^{q} C_{2}\left(\left(\frac{b}{a}\right)^{q / 2}, s\right)\right.\right. \\
& \left.+\left|f^{\prime}(a)\right|^{q} C_{3}\left(\left(\frac{b}{a}\right)^{q / 2}, s\right)\right]^{1 / q} \\
& +b\left[\left|f^{\prime}(\sqrt{a b})\right|^{q} C_{2}\left(\left(\frac{a}{b}\right)^{q / 2}, s\right)\right. \\
& \left.\left.+\left|f^{\prime}(b)\right|^{q} C_{3}\left(\left(\frac{a}{b}\right)^{q / 2}, s\right)\right]^{1 / q}\right\} .
\end{aligned}
$$

Corollary 25. Let the assumptions of Theorem 19 hold. If $\left|f^{\prime}(x)\right| \leq M$ for all $x \in[a, b]$ and $\lambda=0$, then from inequality (29), one gets the following Ostrowski type inequality for fractional integrals:

$$
\begin{aligned}
& \mid\left[\left(\ln \frac{x}{a}\right)^{\alpha}+\left(\ln \frac{b}{x}\right)^{\alpha}\right] f(x)-\Gamma(\alpha+1) \\
& \cdot\left[J_{x-}^{\alpha} f(a)+J_{x+}^{\alpha} f(b)\right] \mid \leq M\left(\frac{1}{\alpha p+1}\right)^{1 / p} \\
& \cdot\left\{a\left(\ln \frac{x}{a}\right)^{\alpha}\left[C_{2}\left(\left(\frac{x}{a}\right)^{q}, s\right)+C_{3}\left(\left(\frac{x}{a}\right)^{q}, s\right)\right]^{1 / q}\right. \\
& +b\left(\ln \frac{b}{x}\right)^{\alpha} \\
& \left.\cdot\left[C_{2}\left(\left(\frac{x}{b}\right)^{q}, s\right)+C_{3}\left(\left(\frac{x}{b}\right)^{q}, s\right)\right]^{1 / q}\right\}
\end{aligned}
$$

for each $x \in[a, b]$.

\section{Competing Interests}

The authors declare that there are no competing interests regarding the publication of this paper.

\section{References}

[1] W. W. Breckner, "Stetigkeitsaussagen für eine Klasse verallgemeinerter konvexer funktionen in topologischen linearen Räumen," Publications de l'Institut Mathématique, vol. 23, pp. 13-20, 1978.

[2] A. A. Kilbas, H. M. Srivastava, and J. J. Trujillo, Theory and Applications of Fractional Differential Equations, Elsevier, 2006.

[3] M. Alomari, M. Darus, S. S. Dragomir, and P. Cerone, "Ostrowski type inequalities for functions whose derivatives are s-convex in the second sense," Applied Mathematics Letters, vol. 23, no. 9, pp. 1071-1076, 2010.

[4] M. Avci, H. Kavurmaci, and M. E. Özdemir, "New inequalities of Hermite-Hadamard type via s-convex functions in the second sense with applications," Applied Mathematics and Computation, vol. 217, no. 12, pp. 5171-5176, 2011.

[5] S. S. Dragomir and S. Fitzpatrik, "The Hadamard's inequality for $s$-convex functions in the second sense," Demonstratio Mathematica, vol. 32, no. 4, pp. 687-696, 1999.

[6] İ. İşcan, "New estimates on generalization of some integral inequalities for s-convex functions and their applications," International Journal of Pure and Applied Mathematics, vol. 86, no. 4, pp. 727-746, 2013.

[7] J. Park, "Generalization of some Simpson-like type inequalities via differentiable $s$-convex mappings in the second sense," International Journal of Mathematics and Mathematical Sciences, vol. 2011, Article ID 493531, 13 pages, 2011.

[8] E. Set, "New inequalities of Ostrowski type for mapping whose derivatives are s-convex in the second sense via fractional integrals," Computers \& Mathematics with Applications, vol. 63, no. 7, pp. 1147-1154, 2012.

[9] M. Z. Sarıkaya, E. Set, and M. E. Özdemir, "On new inequalities of Simpson's type for s-convex functions," Computers \& Mathematics with Applications, vol. 60, no. 8, pp. 2191-2199, 2010. 
[10] M. Z. Sarıkaya, E. Set, H. Yaldız, and N. Başak, "HermiteHadamard's inequalities for fractional integrals and related fractional inequalities," Mathematical and Computer Modelling, vol. 57, no. 9-10, pp. 2403-2407, 2013.

[11] C. P. Niculescu, "Convexity according to the geometric mean," Mathematical Inequalities \& Applications, vol. 3, no. 2, pp. 155$167,2000$.

[12] C. P. Niculescu, "Convexity according to means," Mathematical Inequalities \& Applications, vol. 6, no. 4, pp. 571-579, 2003.

[13] Y. Shuang, H.-P. Yin, and F. Qi, "Hermite-Hadamard type integral inequalities for geometric-arithmetically s-convex functions," Analysis, vol. 33, no. 2, pp. 197-208, 2013.

[14] J. Hua, B.-Y. Xi, and F. Qi, "Hermite-Hadamard type inequalities for geometric-arithmetically s-convex functions," Communications of the Korean Mathematical Society, vol. 29, no. 1, pp. 51-63, 2014.

[15] İ. İscan, "Hermite-Hadamard type inequalities for GA-s-convex functions," Le Matematiche, vol. 69, no. 2, pp. 129-146, 2014.

[16] M. Kunt and İ. İşcan, "On new inequalities of HermiteHadamard-Fejer type for GA-s-convex functions via fractional integrals," Konuralp Jurnal of Mathematics, vol. 4, no. 1, pp. 130139, 2016.

[17] S. Maden, S. Turhan, and İ. İşcan, "New Hermite-HadamardFejer type inequalities for GA-convex functions," in Proceedings of the AIP Conference, vol. 1726, Antalya, Turkey, April 2016.

[18] X.-M. Zhang, Y.-M. Chu, and X.-H. Zhang, "The HermiteHadamard type inequality of GA-convex functions and its application," Journal of Inequalities and Applications, vol. 2010, Article ID 507560, 11 pages, 2010.

[19] T.-Y. Zhang, A.-P. Ji, and F. Qi, "Some inequalities of HermiteHADamard type for GA-convex functions with applications to means," Le Matematiche, vol. 68, no. 1, pp. 229-239, 2013.

[20] J. Wang, J. Deng, and M. Fečkan, "Exploring s-e-condition and applications to some Ostrowski type inequalities via Hadamard fractional integrals," Mathematica Slovaca, vol. 64, no. 6, pp. 1381-1396, 2014.

[21] İ. İşcan, "New general integral inequalities for quasi-geometrically convex functions via fractional integrals," Journal of Inequalities and Applications, vol. 2013, article 491, 15 pages, 2013. 


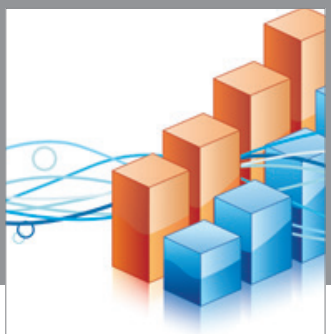

Advances in

Operations Research

vatem alat4

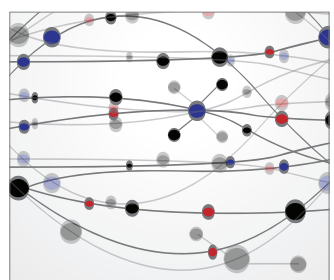

\section{The Scientific} World Journal
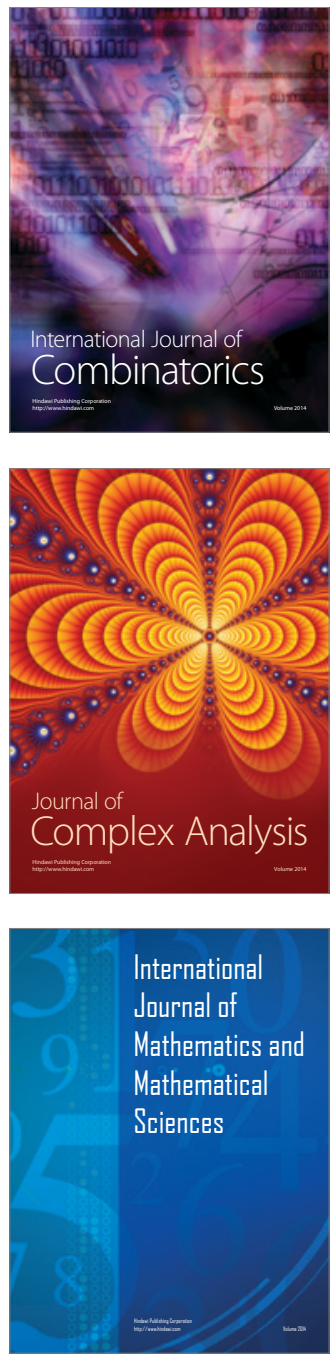
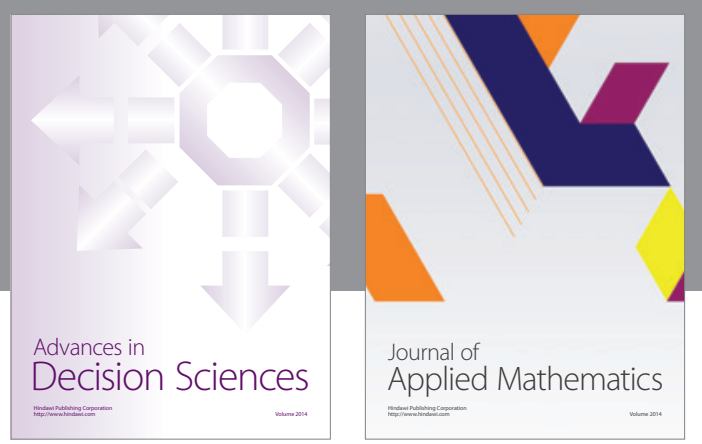

Algebra

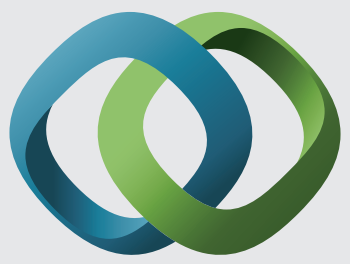

\section{Hindawi}

Submit your manuscripts at

http://www.hindawi.com
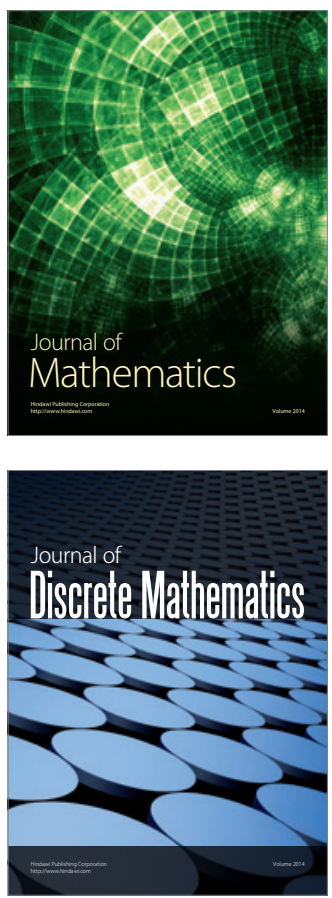

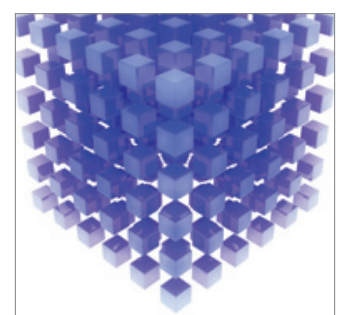

Mathematical Problems in Engineering
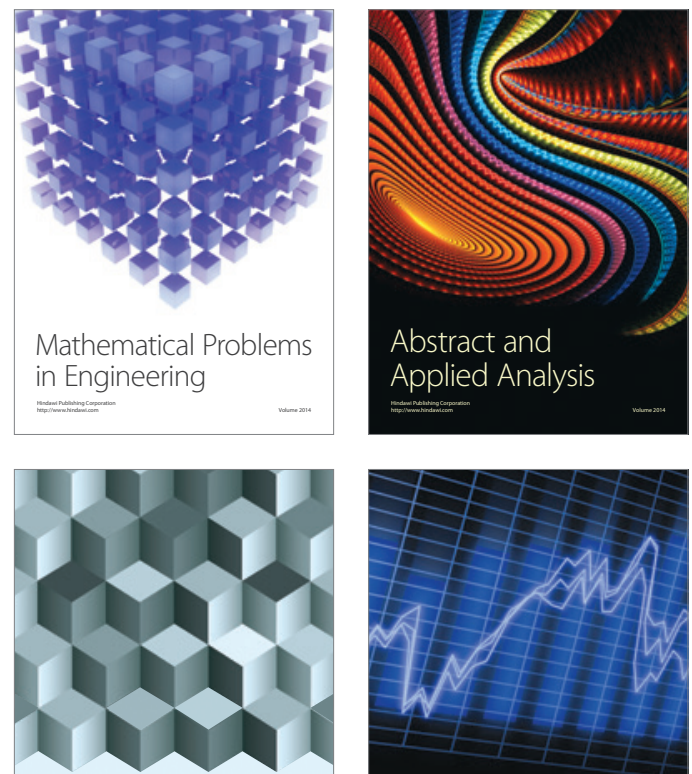

Journal of

Function Spaces

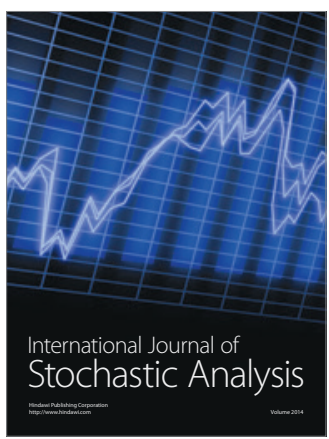

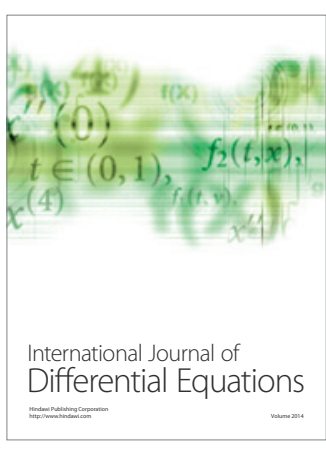
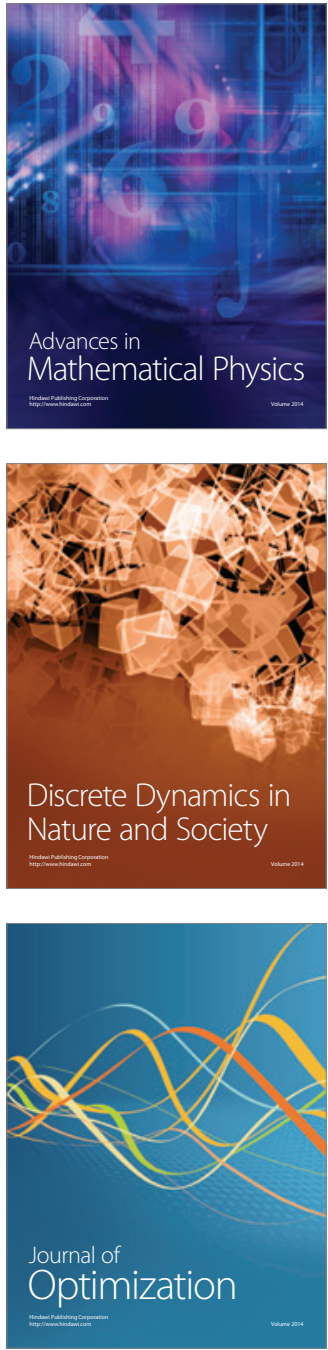